\title{
Nuclear Fusion Driven by Coulomb Explosions of Deuterium Clusters
}

J. Zweiback, R. A. Smith, T. E. Cowan, G. Hays, J. Hartley, R. Howell, C. A. Steinke, K. B. Wharton, T. Ditmire

This article was submitted to

XXVI European Conference on Laser Interaction with Matter 2000 Czech, Republic

June 12-16, 2000

U.S. Department of Energy

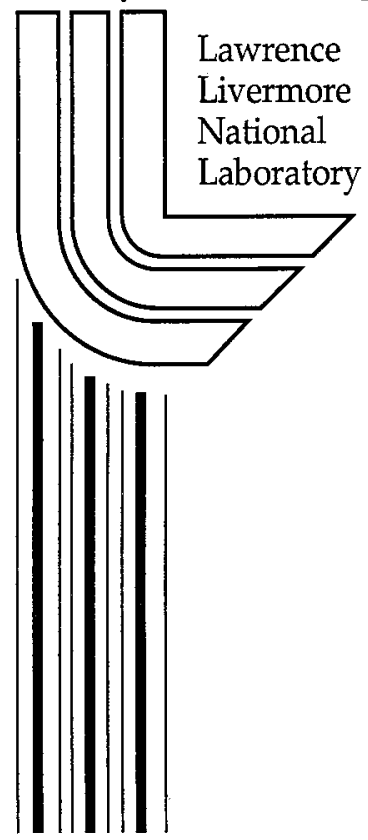

June 9, 2000 


\section{DISCLAIMER}

This document was prepared as an account of work sponsored by an agency of the United States Government. Neither the United States Government nor the University of California nor any of their employees, makes any warranty, express or implied, or assumes any legal liability or responsibility for the accuracy, completeness, or usefulness of any information, apparatus, product, or process disclosed, or represents that its use would not infringe privately owned rights. Reference herein to any specific commercial product, process, or service by trade name, trademark, manufacturer, or otherwise, does not necessarily constitute or imply its endorsement, recommendation, or favoring by the United States Government or the University of California. The views and opinions of authors expressed herein do not necessarily state or reflect those of the United States Government or the University of California, and shall not be used for advertising or product endorsement purposes.

This is a preprint of a paper intended for publication in a journal or proceedings. Since changes may be made before publication, this preprint is made available with the understanding that it will not be cited or reproduced without the permission of the author.

This report has been reproduced

directly from the best available copy.

Available to DOE and DOE contractors from the Office of Scientific and Technical Information

P.O. Box 62, Oak Ridge, TN 37831

Prices available from (423) 576-8401 http://apollo.osti.gov/bridge/

Available to the public from the National Technical Information Service U.S. Department of Commerce 5285 Port Royal Rd., Springfield, VA 22161

http://www.ntis.gov/

OR

Lawrence Livermore National Laboratory Technical Information Department's Digital Library http://www.llnl.gov/tid/Library.html 


\title{
Nuclear fusion driven by Coulomb explosions of deuterium clusters J. Zweiback ${ }^{1}$, R.A. Smith ${ }^{2}$, T.E. Cowan ${ }^{1}$, G. Hays ${ }^{1}$, J.Hartley ${ }^{1}$, R. Howell ${ }^{1}$, C.A. Steinke ${ }^{3}$,K.B.Wharton ${ }^{1}$ and T.Ditmire ${ }^{1}$

\author{
${ }^{1}$ Lawrence Livermore National Laboratory, L-477, Livermore, CA 94550 USA \\ ${ }^{2}$ Imperial College of Science Technology and Medicine, London, SW7 UK \\ ${ }^{3}$ Harvard University, Cambridge, MA 02138, USA
}

\begin{abstract}
We have examined the interaction of deuterium clusters with high intensity, ultrafast laser radiation. Upon irradiation a hot plasma is created with a sufficient temperature to produce nuclear fusion. We have seen that larger clusters produce more fusion neutrons than smaller clusters, consistent with a Coulomb explosion model. Fusion yield is currently limited by propagation effects. Using interferometric imaging we have examined the laser propagation and found that the laser energy is absorbed before it penetrates to the center (highest density region) of the gas jet.
\end{abstract}

\section{INTRODUCTION}

In the past years there have been many experiments studying the nature of intense light interactions with atomic and molecular clusters of a few hundred to a few hundred thousand atoms. These studies have shown that the interaction of laser pulses with pulse width between 0.1 and 2 ps focused to intensity of $10^{16}-10^{17} \mathrm{~W} / \mathrm{cm}^{2}$ with these clusters is extremely energetic. [1-4] Such atomic clusters can be formed from the atoms or molecules in a gas via van der Waals forces. When a high pressure gas expands from a gas jet into vacuum, the cooling associated with the gas's adiabatic expansion can be sufficient to cause nucleation of the gas particles into clusters. The absorption of high intensity laser light by these clusters is many orders of magnitude more efficient than the absorption by single atoms or small molecules in a gas. This is due to the fact that, although the average density in a gas containing clusters is modest $\left(<10^{20} \mathrm{~cm}^{-3}\right)$ the local density within the cluster is high (near solid density of $\sim 10^{23} \mathrm{~cm}^{-3}$ ). As a result, ionization of the cluster material by the intense laser pulse creates free electrons which can be heated by subsequent inverse bremsstrahlung [4]. If the pulse is shorter than the disassembly time of the inertially confined cluster, a very large amount of the laser energy can be deposited in the small spherical microplasma created by ionization of the cluster. A violent explosion of the heated cluster results.

Past experiments have shown that nearly $100 \%$ of the incident energy can be absorbed by the cluster target $[5,6]$. Though the majority of the laser energy is deposited into the electrons within the cluster via inverse bremsstrahlung during the laser pulse, the charge separation of these hot electrons accelerates the ions radially, and, as a result, after the explosion of the cluster the majority $(>90 \%)$ of the laser energy deposited in the cluster is transferred to the ions. Ion energies up to 1 $\mathrm{MeV}$ have been measured in Xe [7].

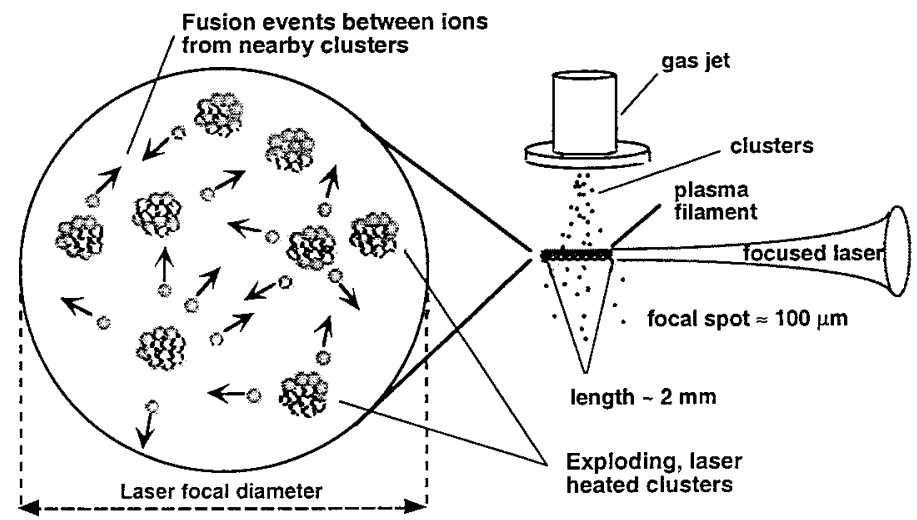

Figure 1: Schematic of the deuterium cluster fusion experiment.

A straightforward extension of this work is to irradiate deuterium clusters in an attempt to create deuterons with enough energy to fuse (greater than a few $\mathrm{keV}$ ). Recently we performed the experiment illustrated schematically in Fig. 1 [8]. In this experiment, a high intensity, ultrafast laser pulse was focused into a gas of deuterium clusters and rapidly heats them. These clusters subsequently explode, ejecting deuterium ions with energies of many $\mathrm{keV}$. This process creates a plasma filament with a diameter of roughly 200 $\mu \mathrm{m}$ and a length of about $2 \mathrm{~mm}$ is length. The fast deuterium ions ejected from the exploding clusters can then collide with ions ejected from other clusters in the plasma. Interferometric images indicates that almost $5 \mathrm{keV}$ of energy per ion is

deposited in the plasma on a fast $(<100 \mathrm{fs})$ time scale, more than sufficient to drive nuclear fusion. Figure 2 shows the energy spectrum of the neutrons produced from this process at several distances from the target. The well known signature of $\mathrm{DD}$ fusion, arising from one branch of the reaction, $\mathrm{D}+\mathrm{D} \rightarrow \mathrm{He}^{3}+\mathrm{n}$, is clearly visible at $2.45 \mathrm{MeV}$. 
Neutron energy (MeV)
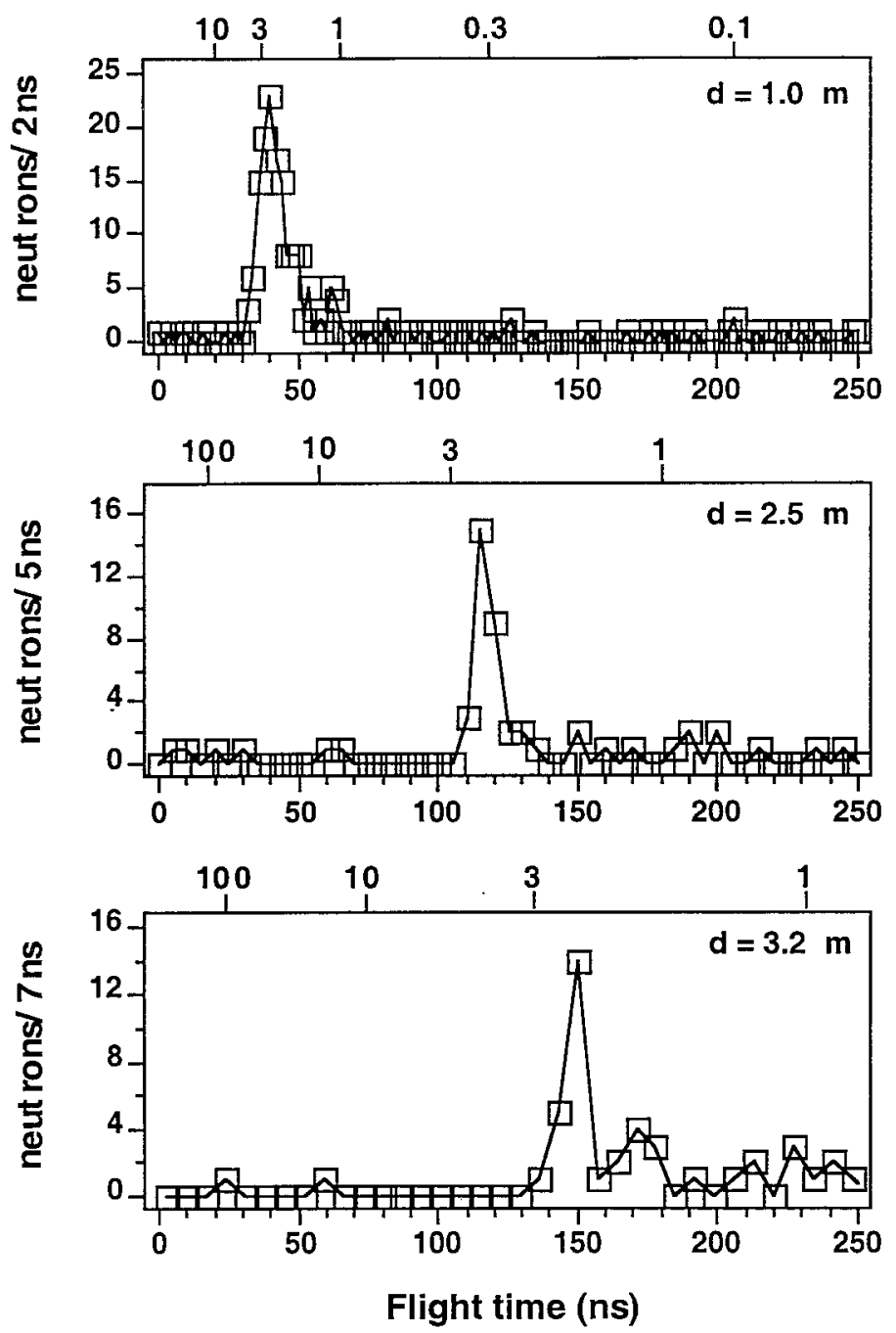

Figure 2: The time-of-flight spectrum of particles detected on the neutron detectors for three plasma-detector separations (1,2.5 and $3.2 \mathrm{~m}$ ). Particle flight time is shown on the bottom axes. The energy of a neutron corresponding to the flight time is shown along the top axes. The presence of $2.45 \mathrm{MeV}$ neutrons is clearly visible in all these spectra.

\section{CLUSTER SIZE EFFECTS}

While large, high $Z$, clusters exhibit a plasmalike behavior $[9,10]$ due to the fact that space charge forces confine the majority of electrons to the cluster, recent experiments and modeling indicate that the deuterium clusters used in these fusion experiments (a few thousand atoms) are dominated by Coulomb explosion behavior. The laser pulse ionizes the cluster and strips away the electrons. The charged sphere will now disassemble via Coulomb explosion. The energy of ions ejected from a Coulomb explosion increases rapidly as the radius of a cluster gets larger. Since the fusion cross-section is highly non-linear with ion energy, an increase in radius can lead to a large increase in neutron yield.

To ascertain the validity of this hypothesis we have undertaken an experiment to explore the scaling of fusion yield with cluster size[11]. Using the well know fact that cluster size increases with decreasing gas jet reservoir temperature, we were able to measure the neutron yield as a function of cluster size by varying gas jet cooling. The results of these experiments are shown in Fig. 3. We find that as the cluster diameter increases from $30 \AA$ to $50 \AA$ there is a large increase in neutron production. The solid line shows a simple model calculation based on the Coulomb explosion picture which agrees well with the data in this size range.

\section{PROPAGATION EFFECTS}

Above $50 \AA$ the data in Fig. 3 begins to deviate from the model and eventually, as the cluster size gets larger, the yield begins to decrease. We wanted to investigate the cause of this discrepancy. The first indication that propagation effects are causing the problem is seen in the Fig.3. It was found that the position of the gas jet relative to the laser focus was important for maximizing the neutron yield. One wanted the laser to come to a focus before the center of the gas jet. The circles are data taken when the jet position was optimized for liquid nitrogen temperatures. In this position the neutron yield begins to decrease once the size gets over $50 \AA$. The squares represent data taken with the jet position re-optimized for liquid helium temperatures. By just moving the position of the jet we were able to obtain about a factor of 2 increase in neutron yield

To examine this further we performed interferometry on the fusion plasma. Using a pellicle to split off a small portion of the incident laser beam, the probe beam was sent across the plasma transverse to the main laser beam. The plasma was then imaged into a shearing Michelson interferometer. The resultant interferograms (an example is seen in Fig. 4) could then be Abel inverted to produce an electron density. In deuterium, provided there is enough energy to fully ionized all atoms, the peak electron density will be the same as the ion energy.

In Fig. 4 one can see an interferogram and the electron density of the plasma as a function distance. The average diameter of the clusters here was approximately $50 \AA$. The electron density increases as one gets closer to the center of the gas jet. This is simply due to the increasing ion density towards the center of the plume. However, $1 \mathrm{~mm}$ before the center of the jet region is reached, the electron density levels off and then begins to decrease rapidly. The laser energy is absorbed before it can reach the highest density regions of the jet. This problem will worsen as the clusters are made larger, becoming 
better absorbers [5]. As this happens the fusion plasma will form further in front of the gas jet at a lower density. The scaling of fusion yield with density $\left(\sim n-{ }^{2}\right)$ will cause the decrease in neutron production seen in Fig. 3.

There are two ways to overcome this problem. The most straightforward way is to increase the laser energy on target. The increased energy will be able to "burn through" the front of the plume, delivering energy to the highest density regions. Alternatively, one could design a supersonic gas nozzle. The current sonic nozzle sprays gas in a very broad plume. A supersonic nozzle would produce a narrow cone and hence a higher density gradient. This would allow more energy to reach the higher density region.

\section{CONCLUSION}

We have produced approximately $10^{4}$ neutrons per shot by irradiating deuterium clusters with an ultrafast laser. We find that cluster size, controlled through reservoir temperature, dramatically effects neutron production in the deuterium fusion. This fusion yield enhancement is due primarily to the greater ion energies produced in the Coulomb explosion of larger clusters. The scaling of the fusion yield, up to a cluster size of $\sim 50 \AA$ is roughly consistent with a simple model for the Coulomb explosion. Above this, size propagation effects limit the neutron yield. The laser energy is absorbed before it can reach the maximum density of the gas jet. Since the high intensity laser does not reach the higher density regions, neutron yield is limited. This problem can likely be overcome with more laser energy and better jet design.

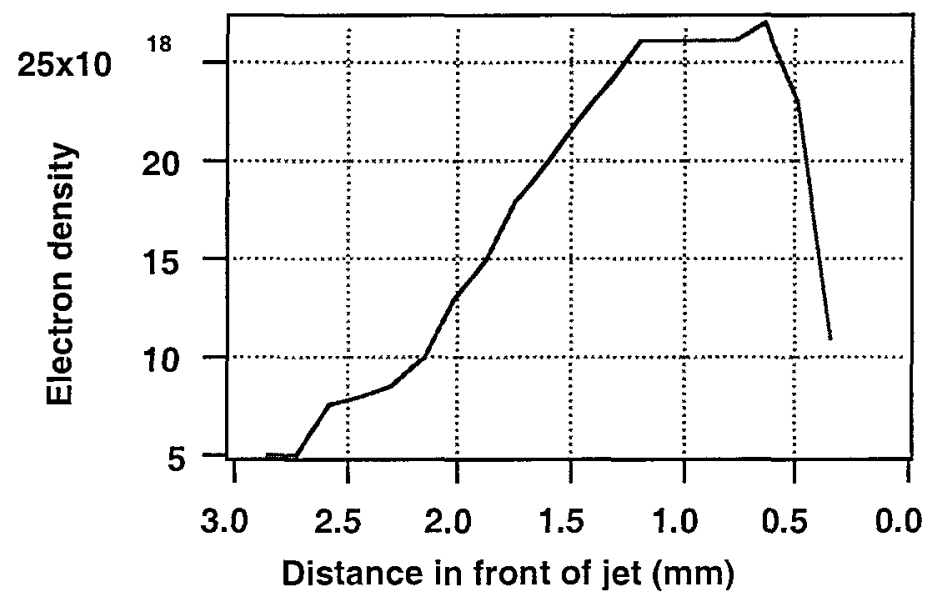

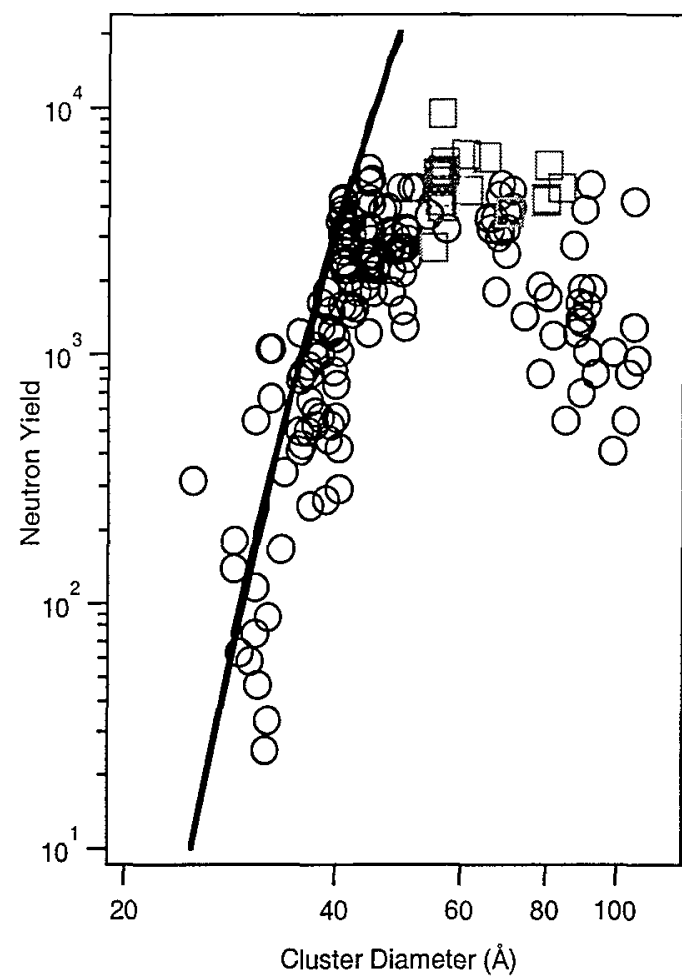

Figure 3: Experimental measurement of neutron yield as a function of cluster size. The circles represent data taken at the same jet location. By moving the jet (square markers) the yield can be increase. This is caused by propagation effects as the laser energy is depleted traversing the target.

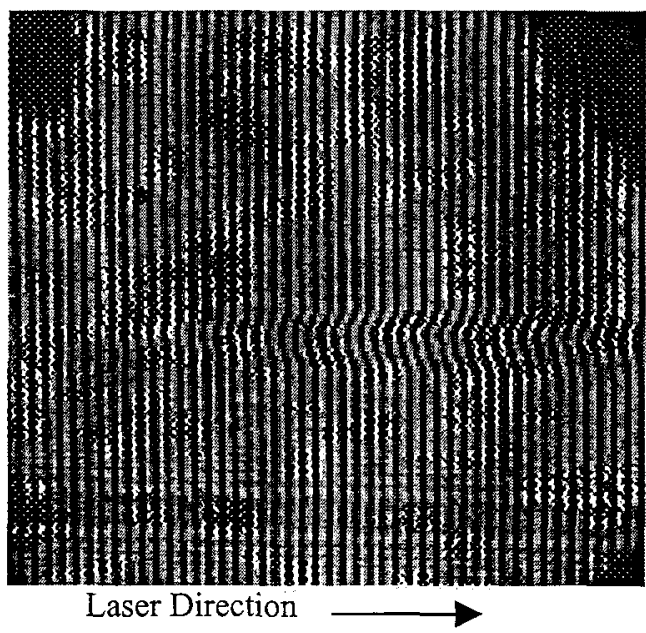

Gas Jet

Figure 4. Abel inversion (left) of interferometric plasma image(right) taken $\sim 20 \mathrm{ps}$ after the laser pulse. The laser pulse is absorbed before it reaches the center of the jet. Full interferogram is $2.8 \mathrm{~mm}$ across. 


\section{ACKNOWLEDGEMENTS}

This work was conducted under the auspices of the U.S. Dept. of Energy under contract W-7405-Eng-48. We would like to acknowledge useful conversations with John Perkins, Tomas Diaz de la Rubia, Howard Powell, Dennis Slaughter and Steve Zinkle. We also acknowledge the important technical assistance of Vince Tsai, Rich Shuttlesworth and Gerry Anderson.

\section{REFERENCES}

1. McPherson, A., Thompson, B. D., Borisov, A. B., Boyer, K., and Rhodes, C. K., "Multiphoton-induced X-Ray Emission at 4-5 keV from Xe Atoms with Multiple Core Vacancies," Nature 370, 631-634 (1994).

2. T. Ditmire, T. Donnelly, A. M. Rubenchik, R. W. Falcone, and M. D. Perry, "The Interaction of Intense Laser Pulses with Atomic Clusters," Phys. Rev. A 53, 3379 (1996).

3. Y.L. Shao, T. Ditmire, J. W. G. Tisch, E. Springate, J.P. Marangos, M.H.R. Hutchinson "Multi-keV Electron Generation in the Interaction of Intense Laser Pulses with Xe Clusters," Phys. Rev. Lett. 77, 3343-3346 (1996).

4. M. Lezius, S. Dobosz, D. Normand, and M. Schmidt, "Explosion Dynamics of Rare Gas Clusters in Strong Laser Fields," Phys. Rev. Lett. 80, 261 (1998).

5. T. Ditmire, R. A. Smith, J. W. G. Tisch and M. H. R. Hutchinson, "High Intensity Laser Absorption in Gases of Atomic Clusters," Phys. Rev. Lett. 78, 3121 (1997).

6. K.Kondo, A.B. Borisov, C. Jordan, A. McPherson, W.A. Schroeder, K. Boyer, and C.K. Rhodes, "Wavelength dependence of multiphoton-induced Xe(M) and Xe(L) emissions from Xe clusters," J. Phys. B 30, 2707 (1997).

7. T. Ditmire, J. W. G. Tisch, E. Springate, M. B. Mason, N. Hay, R. A. Smith, J. P. Marangos and M. H. R. Hutchinson, "High Energy Ions Produced in Explosions of Super-Heated Atomic Clusters," Nature (London), 386, 54 (1997).

8. T. Ditmire J. Zweiback, V. P. Yanovsky, T. E. Cowan, G. Hays, and K. B. Wharton, "Nuclear Fusion from Explosions of Femtosecond-Laser Heated Deuterium Cluster," Nature (London) 398, 489 (1999).

9. J. Zweiback, T. Ditmire, and M. D. Perry, "Femtosecond Time-Resolved Studies of Noble Gas Cluster Explosion Dynamics," Phys. Rev. A. 59, R3166 (1999).

10. L. Köller, M. Schumacher, J. Köhn, S. Teuber, J. Tiggesbäumker, and K.H. Meiwes-Broe, "Plasmon-Enhanced MultiIonization of Small Metal Clusters in Strong Femtosecond Laser Fields," Phys. Rev. Lett. 82, 3783 (1999).

11. J. Zweiback, R.A. Smith T.E. Cowan G. Hays, K.B. Wharton, V.P. Yanovsky, T. Ditmire, "Nuclear Fusion Driven by Coulomb Explosions of Large Deuterium Clusters," PRL 84, 2634 (2000).

This work was performed under the auspices of the U.S. Department of Energy by the University of California, Lawrence Livermore National Laboratory under Contract No. W-7405-Eng-48. 


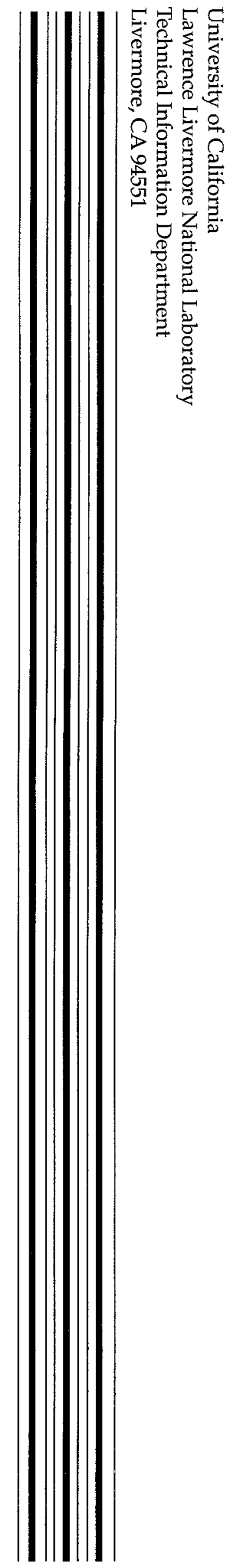

